\title{
Depresión infantil: una revisión bibliométrica
}

\author{
Childhood depression: a bibliometric review
}

DOI: 10.33539/consensus.2019.v24n2.2326

Yin Victoria Zukha Graham Wetzell ${ }^{1}$

\section{RESUMEN}

Este trabajo presenta una revisión de la literatura sobre la depresión infantil (DI) durante el periodo de 2010 hasta 2018. El método empleado ha sido el análisis de documentos sobre esta temática, los cuales fueron identificados en las bases de datos de PROQUEST, EBSCO, artículos de Google académico y libros digitales. Con la información obtenida se realizó un análisis de forma y contenido, revisando además los siguientes conceptos: depresión, depresión infantil y factores que la provocan. De esta manera, se ha encontrado que la investigación sobre este tema, en el periodo señalado líneas arriba, es aún escasa, sobre todo en el ámbito nacional, por lo cual se ve necesario promover la investigación de la DI en Perú desde los diferentes campos de estudios.

\section{Palabras clave}

Depresión, depresión infantil, niños, psicología, factores de riesgo

\begin{abstract}
This work presents a review of the literature about childhood depression (CD), during the period from 2010 to 2018. The used method has been the analysis of documents on this subject, which were identified in the ProQuest, EBSCO databases, articles of Google academic and digital books. Aform and content analysis was conducted with the information obtained, also reviewing the following concepts: depression, childhood depression and factors that cause it. In this way, it has been found that the research about this subject, in the period indicated above, is still limited, especially at the national level, which is why it is necessary to promote the research of childhood depression in Peru from different fields of studies.
\end{abstract}

\section{Keywords}

Depression, childhood depression, children, psychology, factors of risk

1 Licenciada en Educación Primaria, Licenciada en Ciencias Religiosas. Alumna de Posgrado de la Universidad Femenina del Sagrado Corazón. 


\section{INTRODUCCIÓN}

No hay mayor anhelo para toda persona que la búsqueda y consecución de la felicidad. Si esta búsqueda se encuentra en todo ser humano, más aún en los niños que están en pleno desarrollo evolutivo. Sin embargo, la obtención de esta felicidad se ve afectada por una serie de factores que provocan una sintomatología propia de la depresión, la que según los últimos estudios científicos está más presente en la etapa infantil.

De acuerdo con Paelecke - Haberman, Phol y Leplow (2005) la depresión es un trastorno del estado de ánimo que produce malestar psicológico. Tradicionalmente, el término depresión se utilizaba solo para referirse al cuadro clínico que presentaban los adultos, y "aunque en épocas pasadas se rechazaba que los niños sufrieran depresión, actualmente se acepta la existencia de este trastorno en la infancia" (Méndez, 2002, p. 54). Sin embargo, la configuración del concepto de depresión infantil es todavía más reciente; la comunidad científica se mostraba resistente a admitirlo hasta los años setenta. Sin embargo, se han producido cambios significativos en la comprensión del término (Del Barrio, 2010). Así, por primera vez en el IV Congreso Europeo de Paidopsiquiatría, se admite la depresión infantil como un desorden mental (Ramírez, 2009).

El psicólogo y psiquiatra Méndez (2002) expresó: "Un niño deprimido es un ser humano que desde muy temprano apenas sonríe, le falta la ilusión de vivir, la confianza consigo mismo, la capacidad para hacer y conservar las amistades" (p. 15). Al respecto, los estudios revisados manifiestan que, si un niño ha presentado la sintomatología depresiva, hay más probabilidades que en su adultez la desarrolle frente a otros eventos. Por eso, es importante que se reconozcan bien los rasgos más representativos de un niño depresivo y los factores por los que presenta dicho trastorno.

Se eligió el tema sobre la depresión infantil para profundizar en este campo, ya que se está observando una mayor frecuencia en edades preescolar y escolar. Otro motivo por el que se seleccionó el presente tema es debido a que constituye el primer problema de salud pública en el Perú, según Huamanttica (2016) y es necesario revalorizar el abordaje del asunto en beneficio de la niñez que está expuesta a este desorden mental del que nadie está exento y que muchas veces pasa desapercibido.

Los síndromes depresivos en niños han sido complejos en su diagnóstico. Muchas veces se ha comparado el cuadro que se presenta en adultos debido a que, en los niños, las manifestaciones clínicas han sido diferentes. Esto ha generado dificultades para detectar cuándo un niño está deprimido. Incluso, los mismos adultos de su entorno no lo suelen notar. Una evaluación realizada a un niño puede presentar escalas de puntaje bajo, sin embargo, otras evidencias muestran que la depresión está presente en el menor. Por ello, es recomendable evaluar de acuerdo al nivel de comprensión y etapa evolutiva del menor, utilizando entrevistas semiestructuradas y la observación directa (Pinto y Álvarez, 2010).

El objetivo del presente artículo es que, a partir de una revisión en algunas bases de datos y referencias bibliográficas digitales, se recopile los estudios que se han realizado 
sobre la depresión infantil a nivel nacional e internacional, así como analizar los factores que la provocan predominantemente.

La Organización Mundial de la Salud (OMS, 2004, citado por Robles, Sánchez, y Galicia, 2011) ha estimulado en los últimos años una prioridad por la investigación, prevención y tratamiento de las enfermedades mentales. Ahora bien, citando a Medina-Mora et al. -2003-; OMS -2011- y a Pardo, Sandoval y Umbarita -2004-, Robles, Sánchez y Galicia afirman que, de acuerdo con la OMS, entre las enfermedades mentales que destacan se encuentra la depresión, siendo la cuarta causa de morbilidad y, que a partir del año 2020 será la segunda causa.

El Manual Diagnóstico y Estadístico de los Trastornos Mentales (Diagnostic and Statistical Manual of Mental Disorders, abreviado DSM) define un episodio depresivo mayor como un síndrome, en el que deben estar presentes al menos 5 síntomas durante un periodo de 2 o más semanas. La Asociación de Psiquiatría de los Estados Unidos (American Psychiatric Association) establece estos síntomas como característicos de un cuadro de depresión en niños y adolescentes (ver tabla 1). En el caso de los niños, el énfasis se presenta en la disforia y la irritabilidad (Pérez et al., 2011).

Los síntomas más frecuentes son:

- Afectivos: tristeza, apatía, ansiedad, etc.

- Motivacionales: poca fuerza de voluntad, deseos de suicidio, etc.

- Cognitivos: problemas de atención, dificultades de memoria, etc.

- Motores: pasividad, lentitud, agitación, etc.

- Físicos: insomnio, pérdida de apetito, cansancio.

\section{Tabla 1}

Sintomas del síndrome depresivo mayor en niños y adolescentes

- Estado de ánimo triste o irritable.

- Pérdida persistente del interés o de la capacidad de disfrute por las actividades favoritas.

- Alteraciones en los patrones de sueño.

- Modificaciones en el peso o en el apetito y falta en la ganancia de peso.

- Disminución en la capacidad de razonamiento o concentración.

- Preocupación o ideación suicida.

- Alteraciones psicomotoras como: agitación o retardo.

- Fatiga o pérdida de energía.

- Sentimiento de inutilidad o culpa.

Síntomas del Síndrome Depresivo en Niños y adolescentes. La Asociación de Psiquiatría de Estados Unidos (American Psychiatric Association), ha establecido que deben estar presentes cinco o más de los síntomas mencionados durante el periodo de dos o más semanas para clasificar a un paciente pediátrico con un episodio depresivo.

Fuente: Pérez, Rivera, Soto-Vega y Muñoz-Pérez (2011, p. 152). 
Los factores que influyen en el desarrollo de la depresión infantil son diversos, pero por cuestiones de orden y a raíz de las revisiones, se encontró que muchas investigaciones los clasifican de diversas maneras. En esta oportunidad se ha tenido en cuenta la clasificación de Méndez (2011, citada por Ortega, 2015) en la revisión que efectuó sobre los factores de riesgo y protección de la depresión infantil. Por lo tanto, afirmar que un solo factor interviene en el desarrollo de la depresión infantil sería casi insostenible, puesto que, el niño está en constante interacción con su entorno. Por ello, los factores se afectan unos a otros, siendo la DI el resultado de la combinación de ellos. Como lo muestra la tabla 2 de Méndez (2011, citada por Ortega, 2015, p.13), los niños pueden adquirir mayor o menor riesgo de padecer este síndrome según los factores ambientales y los factores personales.

Tabla 2

Interacción entre factores ambientales y factores personales

\begin{tabular}{lcllll}
\hline Factores ambientales & Interacción & Factores personales & Resultado & Riesgo depresión infantil \\
\hline Ambiente muy negativo & $\mathrm{X}$ & Niño muy vulnerable & $=$ & Máximo \\
Ambiente muy negativo & $\mathrm{X}$ & Niño competente & $=$ & Medio \\
Ambiente neutro o algo positivo & $\mathrm{X}$ & Niño muy vulnerable & $=$ & Medio \\
Ambiente muy positivo & $\mathrm{X}$ & Niño competente & $=$ & Mínimo \\
\hline
\end{tabular}

Fuente: Méndez (2011, citado por Ortega, 2015, p.13).

La depresión infantil, al igual que la depresión en el adulto, se relaciona con los desequilibrios en los neurotransmisores como la serotonina, norepinefrina y dopamina, exactamente cuando estos disminuyen (García-Vera y Sanz, 2012). Por otro lado, hay datos que indican que la depresión tiene un componente genético porque en pacientes que la padecen existen antecedentes familiares sujetos a este cuadro.

Los elementos que pueden propiciar que un niño padezca o no depresión se deberán a una serie de recursos en su personalidad como su tipo de temperamento, si es introvertido o extrovertido, su nivel de autoestima y el grado de vulnerabilidad, que hace que ante un mismo evento las consecuencias sean más perjudiciales y prolongadas para unos niños que para otros. También, Méndez
(2011, citado por Ortega, 2015) señala que la DI está relacionada con déficits en la capacidad de resolución de problemas, autoevaluación y autoreforzamiento. Según el Modelo Cognitivo, el niño puede establecer diversas formas de pensamiento negativo acerca de sí mismo e incluso, aunque sus experiencias sean positivas, realiza una errada interpretación de su realidad concreta.

Aquí se encuentran factores como el sexo, la zona de procedencia (rural o urbana), la edad, la raza, entre otras.

Los estudios mencionados con anterioridad indican que la depresión no está sujeta determinantemente por estos factores. Sin embargo, se han realizado estudios con relación a la edad y al sexo. 
Con respecto al sexo, en la etapa de la infancia los niños no presentan mayores diferencias de quien lo padece, es decir, tanto en niñas como en niños existe la misma probabilidad de desarrollar la DI.

De acuerdo con el Grupo de trabajo de la actualización de la Guía de Práctica Clínica sobre la Depresión Mayor en la Infancia y la Adolescencia (2018), en referencia a la edad, en un estudio realizado en Australia con una muestra comunitaria de niños entre
4 - 11 años se encontró que la prevalencia de episodio depresivo mayor, según el DSM - IV era de un $1.1 \%$, prácticamente igual en niños que en niñas. De la misma manera con respecto al género.

El Grupo de trabajo que estuvo a cargo de la actualización de la Guía de Práctica Clínica mencionada anteriormente, muestra en la tabla 3 los factores de riesgo asociados a la depresión en niños y adolescentes.

\section{Tabla 3}

Factores de riesgo asociados a la depresión en niños y adolescentes

\begin{tabular}{|c|c|c|c|}
\hline I & $\begin{array}{l}\text { B } \\
\text { i } \\
\text { o }\end{array}$ & Genéticos & $\begin{array}{l}\text { Historia familiar de depresión o trastorno bipolar. } \\
\text { La información actual sugiere que los factores genéticos podrían ser menos } \\
\text { importantes en la presentación de depresión en la niñez que en la adolescencia. }\end{array}$ \\
\hline $\begin{array}{l}\text { n } \\
\text { d } \\
\text { i }\end{array}$ & $\begin{array}{l}\text { ó } \\
\mathrm{g} \\
\mathrm{i} \\
\mathrm{c}\end{array}$ & $\begin{array}{l}\text { Sexo } \\
\text { Edad }\end{array}$ & $\begin{array}{l}\text { Antes de la pubertad la frecuencia de trastornos depresivos es prácticamente } \\
\text { igual en niños que en niñas; en la adolescencia son entre } 2 \text { y } 3 \text { veces más } \\
\text { frecuentes en el sexo femenino. }\end{array}$ \\
\hline i & $\begin{array}{l}\mathrm{O} \\
\mathrm{s}\end{array}$ & Problemas crónicos de salud & Incluyendo enfermedades médicas y discapacidades físicas o de aprendizaje. \\
\hline d & $\begin{array}{l}\mathrm{P} \\
\mathrm{S} \\
\mathrm{i}\end{array}$ & Otros trastornos psíquicos & $\begin{array}{l}\text { Particularmente trastornos de ansiedad, trastornos de conducta o TDAH. } \\
\text { Disforia de género. }\end{array}$ \\
\hline l & $\begin{array}{l}\mathrm{c} \\
\mathrm{o}\end{array}$ & Orientación sexual & Homosexualidad. \\
\hline $\begin{array}{l}\text { e } \\
\text { s }\end{array}$ & $\begin{array}{l}1 \\
\text { ó } \\
\text { g } \\
\text { i } \\
\text { c } \\
\text { o } \\
\text { S }\end{array}$ & $\begin{array}{l}\text { Temperamento } \\
\text { Afectividad negativa } \\
\text { Otros estilos cognitivos }\end{array}$ & $\begin{array}{l}\text { Tendencia a experimentar estados emocionales negativos, que se acompañan } \\
\text { de características cognitivas (por ej. baja autoestima) y conductuales. } \\
\text { Conlleva una mayor reactividad a estímulos negativos. } \\
\text { Estilo cognitivo rumiativo. } \\
\text { Déficits de habilidades sociales. }\end{array}$ \\
\hline
\end{tabular}

Acontecimientos vitales estresantes Pérdidas u otros acontecimientos traumáticos.

F Trastorno psíquico Especialmente depresión (incluyendo depresión/ ansiedad materna en $\mathrm{m}$ en los padres
A $\mathrm{i}$

m i Conflictos en las

b $\stackrel{\mathrm{r}}{\mathrm{r}}$ relaciones familiares

i e

e $\mathrm{s}$

n Maltrato/Abuso

t

a $\mathrm{S}$

l 0

e $\stackrel{\mathrm{c}}{\mathrm{i}}$

s

e

$\mathrm{s}$

sociales adversas

relaciones con iguales

Acoso (bullying) embarazo y posparto).

Trastorno por uso/abuso de alcohol u otras sustancias.

Separación/divorcio, conflictos conyugales. Conflictos en relaciones paterno-filiales.

Negligencia en cuidados, escasa afectividad o rechazo.

Físico, emocional o sexual.

Mala adaptación o fracaso académico.

Conflictos interpersonales, ausencia de amistades

Incluyendo el trato degradante, la humillación, burla o el rechazo/aislamiento.

Sin hogar, institucionalizados, en centros de acogida o de seguridad. Emigración. Refugiados.

Fuente: Grupo de trabajo de la actualización de la Guía de Práctica Clínica sobre la Depresión Mayor en la Infancia y la Adolescencia (2018, p. 68). 
La familia es el más importante entorno en donde se desarrolla un niño. Mucho de lo que acontece en este medio vital está muy asociado a provocar la DI en la infancia. Kovacs (2010) defiende que el tipo de vínculo de la madre con el niño es lo que puede provocar depresión. Esto quiere decir que la depresión materna estaría muy relacionada a la depresión infantil. Y no solo con la madre, la relación que el niño establezca con los otros miembros de la familia, su idiosincrasia, el modelo de crianza impuesto, las relaciones agresivas entre padres, los continuos conflictos, divorcios, van ocasionando desajustes en el manejo emocional del niño.

La escuela es el segundo medio que tiene mayor influencia en la vida de un niño, puesto que en ella se marcan los procesos de aprendizaje y el niño va reconociendo sus habilidades y capacidades.

Entre los resultados de algunos estudios se mencionan ciertas condiciones negativas que presentan los ambientes físicos de estudio: variación de temperaturas, presencia de ruido excesivo, escasa iluminación y sobrepoblación (Orlandini, 1996, citado por Esparza y Rodríguez, 2009).

La escuela puede presentar eventos estresantes que despierten en el niño niveles altos de frustración, presiones, desaprobación, miedo al castigo, ansiedad, etc. A esto, se añade el rendimiento académico al que se ve presionado por alcanzar altos estándares.

Por otro lado, las relaciones que se establecen en la propia escuela son fundamentales para el desarrollo emocional de los niños. No solo las relaciones con los profesores pueden influir en ellos, sino las relaciones que tengan con sus pares, es decir, con sus compañeros.

Un aspecto importante que presentan los niños depresivos al relacionarse con sus amigos de la escuela es que tienen la tendencia a buscar amigos que presentan también estos síntomas, y eso genera la probabilidad que el cuadro depresivo se prolongue por más tiempo, incluso no sea reconocido (Zalk, Kerr, Branje, Stattin y Meeus, 2010; citados por Del Barrio, 2010, p. 51).

La diversidad de factores de riesgo plantea el cuestionamiento de saber qué factor tiene mayor predominancia entre todos. Uno de los objetivos de este artículo de revisión es valorar el punto crucial de origen de mayor relevancia de la depresión infantil.

\section{METODOLOGÍA}

El método empleado ha sido el análisis de documentos sobre esta temática, para lo cual se consultó la base de datos Proquest y EBSCO. Sin embargo, aunque se ha llevado a cabo una búsqueda detallada en esas bases a las que se tuvo acceso, se ha recurrido también a otras fuentes en Google Académico (libros online, tesis de grado y tesis doctorales, páginas web, artículos de revisión, informes, programas) ya que aportan información relevante sobre la depresión infantil y los factores que provocan este cuadro médico. En la base de datos no se encuentran muchos artículos de los últimos diez años. Los criterios de búsqueda en estas bases de datos, en las que se encuentra más información, se realizó incluyendo el término "depresión", en líneas generales. En Google académico, se hizo una búsqueda detallando el año 
de publicación a partir del 2014. Pese a ello, aparecían publicaciones de fechas anteriores. Al momento de delimitar la búsqueda se utilizó la palabra "depresión", “depresión infantil", "factores de depresión y hubo una variedad de documentos que se tuvo que seleccionar posteriormente. Se descartó los que abordaban temas referidos a la depresión en la adolescencia, y se descargó los documentos que abordaban la depresión infantil, sea desde el ámbito educativo o clínico. Se debe mencionar que en Google académico hay más material en temas de depresión en la adolescencia y sus formas de prevención y tratamiento, en comparación a la depresión presentada en niños.

En una primera fase, se revisaron los documentos que presentaban relación con el eje temático de "Depresión infantil", la conceptualización médica, epidemiología, sintomatología y los factores en sus distintas clasificaciones.

En una segunda fase se tomó en cuenta un estudio realizado en Australia que muestra que la prevalencia de episodio depresivo mayor es de $1.1 \%$ tanto en niños como en niñas, confirmando que el factor personal, en el ámbito demográfico no está condicionado en gran medida por el género en la etapa infantil, a diferencia de las variaciones que si se encuentran en la adolescencia. Así mismo, Costello y otros (2003, citados por Siabato, Forero y Salamanca, 2017) han encontrado en sus estudios sobre la depresión infantil y en adolescentes la mayor prevalencia de este trastorno en las mujeres, alertando cada vez más que, en esta etapa del desarrollo se debe enfocar una intervención oportuna y de prevención en el género femenino porque presenta mayor vulnerabilidad.
En una tercera fase, se encontraron investigaciones que muestran la predominancia de ciertos factores de riesgo sobre otros como el género que, durante la etapa de la adolescencia se presenta con mayor frecuencia en las niñas; el ambiente familiar por sobre las características psicológicas del niño y la condición biológica $\mathrm{y} / \mathrm{o}$ genética por sobre algún problema crónico de salud.

Muchos niños y adolescentes presentan dificultades para mantener una vida sana, dado que las experiencias y otros condicionamientos han generado que actualmente existan más menores con esta discapacidad en su salud mental. La Organización Mundial de la Salud (2014) encuentra a la depresión como la mayor causa de invalidez para sostener una vida equilibrada.

Finalmente, se seleccionaron algunas tablas que presentan la sintomatología y el grado de vulnerabilidad que presentan los niños con relación a factores personales y ambientales.

A través del análisis bibliométrico se pretende conocer la depresión infantil como una realidad existente en el campo de la salud mental de los niños, revalorizando el interés que debe existir por este tema y buscando propiciar mayores investigaciones que analicen cuál es el factor mayormente predominante para que un niño manifieste síntomas de depresión.

\section{RESULTADOS}

Dentro del análisis de la calidad de los artículos revisados la mayoría están registrados dentro del ranking de Scimago predominantemente en el Quartil 4. Del 
mismo modo, se encontró que la mayoría de los documentos revisados tienen indexación en diferentes bases de datos (ver tabla 4).

Se procedió a la revisión contando con 21 documentos, de los cuales 9 son artículos provenientes de diversas revistas. Todas estas se encuentran indexadas, pero solo 9 están presentes en el ranking de Scimago ubicadas dentro de un Quartil. Entre el resto de documentos revisados se halló lo siguiente:

\section{Tabla 4}

Indexación de las revistas revisadas

\begin{tabular}{|c|c|c|c|}
\hline Revista & Base de datos & Contenido & Quartil \\
\hline $\begin{array}{l}\text { Revista Mexicana } \\
\text { de Neurociencia }\end{array}$ & Medigraphic & $\begin{array}{l}\text { Trastorno depresivo mayor en niños } \\
\text { y adolescentes }\end{array}$ & Q4 \\
\hline $\begin{array}{l}\text { Salud Mental } \\
\text {-Instituto } \\
\text { Nacional de } \\
\text { Psiquiatría Ramón } \\
\text { de la Fuente }\end{array}$ & Medigraphic & $\begin{array}{l}\text { Prevalencia de trastornos mentales } \\
\text { y uso de servicios: Resultado } \\
\text { de la Encuesta Nacional de } \\
\text { Epidemiología Psiquiátrica en } \\
\text { México }\end{array}$ & Q4 \\
\hline $\begin{array}{l}\text { Diversitas: } \\
\text { Perspectivas en } \\
\text { Psicología }\end{array}$ & $\begin{array}{l}\text { - Psicodoc } \\
\text { - Scientific Electronic Library Online } \\
\text { - Dialnet } \\
\text { - Directory Of Open Access Journals } \\
\text { - Latindex - Directorio (Sistema } \\
\text { Regional De Información En Línea } \\
\text { Para Revisas Científicas De América } \\
\text { Latina El Caribe, España Y Portugal) } \\
\text { - Índice De La Literatura } \\
\text { Latinoamericana En Ciencias De La } \\
\text { Salud } \\
\text { - Pepsic-Periódicos Eletrônicos Em } \\
\text { Psicologia } \\
\text { - Bvs-Biblioteca Virtual En Salud } \\
\text { - Index Copernicus } \\
\text { - Citas Latinoamericanas En Ciencias } \\
\text { Sociales Y Humanidades } \\
\text { - Índice Bibliográfico Nacional } \\
\text { Publindex - A2 }\end{array}$ & $\begin{array}{l}\text { Factores contextuales del desarrollo } \\
\text { infantil y su relación con los estados } \\
\text { de ansiedad y depresión. }\end{array}$ & Q1 \\
\hline $\begin{array}{l}\text { Pensamiento } \\
\text { Psicológico }\end{array}$ & $\begin{array}{l}\text { - Proquest } \\
\text {-Latindex } \\
\text { - Catálogo (Sistema Regional De } \\
\text { Información En Línea Para Revistas } \\
\text { Científicas De América Latina, El } \\
\text { Caribe, España Y Portugal) } \\
\text { - Scientific Electronic Library Online } \\
\text { - Directory Of Open Access Journals } \\
\text { - Índice De La Literatura } \\
\text { Latinoamericana En Ciencias De La } \\
\text { Salud } \\
\text { - BVS: Biblioteca Virtual En Salud }\end{array}$ & $\begin{array}{l}\text { Asociación entre depresión e } \\
\text { ideación suicida en un grupo de } \\
\text { adolescentes colombianos. }\end{array}$ & Q1 \\
\hline
\end{tabular}




\begin{tabular}{|c|c|c|c|}
\hline & $\begin{array}{l}\text { - Índice Bibliográfico Nacional } \\
\text { Publindex - A2 } \\
\text { - DIALNET } \\
\text {-Clasificación Integrada De Revistas } \\
\text { Científicas-Grupo C } \\
\text { - Qualis/Capes - B4 Homologación }\end{array}$ & & \\
\hline $\begin{array}{l}\text { Revista Tesis } \\
\text { Psicológica }\end{array}$ & $\begin{array}{l}\text { - Latindex - Catálogo (Sistema } \\
\text { Regional De Información En Línea } \\
\text { Para Revistas Científicas De América } \\
\text { Latina, El Caribe, España Y Portugal) } \\
\text { - Dialnet } \\
\text { - Qualis/Capes - C Homologación }\end{array}$ & $\begin{array}{l}\text { Prevalencia de depresión infantil en } \\
\text { colegios oficiales de Barranquilla }\end{array}$ & - \\
\hline $\begin{array}{l}\text { Información } \\
\text { Psicológica }\end{array}$ & $\begin{array}{l}\text { - Psicodoc, Dialnet, Redalyc, Latindex, } \\
\text { Redin, Isoc }\end{array}$ & $\begin{array}{l}\text { La depresión infantil a la altura de } \\
\text { nuestro tiempo. }\end{array}$ & - \\
\hline $\begin{array}{l}\text { Psicología Escolar } \\
\text { y Educacional }\end{array}$ & Scielo & $\begin{array}{l}\text { Depresión en adolescentes. } \\
\text { Relaciones con el desempeño } \\
\text { académico. }\end{array}$ & Q4 \\
\hline $\begin{array}{l}\text { Psicología } \\
\text { Iberoamericana }\end{array}$ & $\begin{array}{l}\text { - Redib } \\
\text { - Dialnet } \\
\text { - Erihplus } \\
\text { - Latindex } \\
\text { - C.I.R.C. } \\
\text { - Miar } \\
\text { - Redalyc }\end{array}$ & $\begin{array}{l}\text { Relación del Bienestar Psicológico, } \\
\text { Depresión y Rendimiento } \\
\text { Académico en Estudiantes de } \\
\text { Secundaria. }\end{array}$ & \\
\hline $\begin{array}{l}\text { Revista } \\
\text { Intercontinental } \\
\text { de Psicología y } \\
\text { Educación }\end{array}$ & - Ebsco & $\begin{array}{l}\text { La sintomatología depresiva y el } \\
\text { desempeño académico. }\end{array}$ & - \\
\hline $\begin{array}{l}\text { Electronic Journal } \\
\text { of Research in } \\
\text { Educational } \\
\text { Psychology }\end{array}$ & - Ebsco & $\begin{array}{l}\text { Relación entre el rendimiento } \\
\text { escolar y la sintomatología } \\
\text { depresiva en niños españoles. }\end{array}$ & Q3 \\
\hline $\begin{array}{l}\text { Revista de } \\
\text { Psicopatología y } \\
\text { Psicología Clínica }\end{array}$ & $\begin{array}{l}\text { - Academic Search Complete (Ebsco) } \\
\text { - Academic Search Premier (Ebsco) } \\
\text { - A360grados } \\
\text { - Compludoc } \\
\text { - C- opac } \\
\text { - Dialnet } \\
\text { - Dice } \\
\text { - Dulcinea } \\
\text { - E-Spacio } \\
\text { - Feap } \\
\text { - Google Académico } \\
\text { - In-Recs } \\
\text { - Isoc (Csic) } \\
\text { - Latindex } \\
\text { - Linceo } \\
\text { - Miar } \\
\text { - Psicodoc } \\
\text { - Psycinfo } \\
\text { - Rcs } \\
\text { - Psyke }\end{array}$ & $\begin{array}{l}\text { Prevención de la depresión en niños } \\
\text { y adolescentes: revisión y reflexión. }\end{array}$ & Q2 \\
\hline
\end{tabular}




\begin{tabular}{|c|c|c|c|}
\hline & $\begin{array}{l}\text { - Pubpsych } \\
\text { - Redib } \\
\text { - Resh } \\
\text { - Scimago Journal Rank } \\
\text { - Scopus } \\
\text { - Sumaris Cbuc } \\
\text { - Ulrich's } \\
\text { - Zdb }\end{array}$ & & \\
\hline $\begin{array}{l}\text { Revista Médica } \\
\text { de Costa Rica y } \\
\text { Centroamérica }\end{array}$ & - Medigraphic & Depresión en niños y adolescentes. & - \\
\hline $\begin{array}{l}\text { Revista Peruana } \\
\text { de Psicología: } \\
\text { Liberabit }\end{array}$ & $\begin{array}{l}\text { - Proquest } \\
\text { - Esci } \\
\text { - Scielo } \\
\text { - Redalyc } \\
\text { - Ebsco } \\
\text { - Psicodoc } \\
\text { - Doa } \\
\text { - Redib } \\
\text { - Dialnet }\end{array}$ & $\begin{array}{l}\text { La investigación de la depresión en } \\
\text { adolescentes en el Perú: una revisión } \\
\text { sistemática. }\end{array}$ & - \\
\hline $\begin{array}{l}\text { Revista } \\
\text { Latinoamericana } \\
\text { de Psicología }\end{array}$ & - Ebsco & $\begin{array}{l}\text { Estilos de personalidad dependiente } \\
\text { y autocrítico: desempeño cognitivo } \\
\text { y sintomatología depresiva. }\end{array}$ & Q2 \\
\hline $\begin{array}{l}\text { International } \\
\text { Journal of } \\
\text { Psychology and } \\
\text { Psychological } \\
\text { Therapy }\end{array}$ & - Dialnet & $\begin{array}{l}\text { Relaciones entre ansiedad escolar, } \\
\text { ansiedad rasgo, ansiedad estado } \\
\text { y depresión en una muestra de } \\
\text { adolescentes españoles. }\end{array}$ & \\
\hline $\begin{array}{l}\text { Revista de } \\
\text { Psicología Clínica } \\
\text { con Niños y } \\
\text { Adolescentes }\end{array}$ & $\begin{array}{l}\text { - Scope } \\
\text { - Scimago }\end{array}$ & $\begin{array}{l}\text { Intervención cognitivo-conductual } \\
\text { en un caso de depresión en una } \\
\text { adolescente tardía. }\end{array}$ & Q1 \\
\hline $\begin{array}{l}\text { Revista de } \\
\text { Psiquiatría } \\
\text { y Psicología } \\
\text { del Niño y del } \\
\text { Adolescente }\end{array}$ & - & $\begin{array}{l}\text { Relación entre las conductas de } \\
\text { intimidación, depresión e ideación } \\
\text { suicida en adolescentes. Resultados } \\
\text { preliminares. }\end{array}$ & \\
\hline Ajayu & & $\begin{array}{l}\text { Terapia Breve y Depresión infantil } \\
\text { (Estudio de Caso). }\end{array}$ & \\
\hline
\end{tabular}

En la revisión realizada, teniendo en cuenta el desarrollo del objetivo del artículo, los documentos han sido divididos en tres bloques:

1) Estudios realizados sobre la depresión infantil a nivel nacional e internacional.
Con respecto al lugar en el que se realizaron mayores investigaciones de DI, se observa en la Tabla 5 que en el ámbito internacional se ha efectuado gran número de investigaciones sobre este tema (93\%) -España, Australia, Colombia, México-, comparado con el nivel nacional $(7 \%)$ -Lima y Trujillo-. 


\section{Tabla 5}

Documentos cuyos contenidos de depresión infantil y otros, se realizaron a nivel nacional e internacional

\begin{tabular}{lrr}
\hline Lugar & $\mathrm{N}^{\mathrm{o}}$ & $\%$ \\
\hline Documentos Nacionales & 2 & 7 \\
Documentos internacionales & 26 & 93 \\
Total & 28 & 100 \\
\hline
\end{tabular}

Fuente: elaboración propia.

2) Factores de riesgo

En la Tabla 6 se observa que en los documentos revisados la depresión infantil ha sido estudiada relacionándola con otros temas. Con un 53\% se deduce que hay mayor investigación sobre artículos relacionados con la sintomatología, así como los factores de riesgo que la provocan. Del mismo modo en el segundo hallazgo más frecuente fueron documentos enfocados a la DI abordándola junto con la adolescencia (18\%).

\section{3) Tratamiento}

Por otro lado, se puede notar que con respecto al tratamiento, terapia o prevención de la depresión infantil hay escasas investigaciones ( $8 \%$; ver tabla 6$)$. También, desde el ámbito psicoeducativo se ha hallado un solo artículo que aborda la DI en el colegio por la cual se registra solo un $3 \%$ en la búsqueda.

Tabla 6

Documentos cuyos contenidos están relacionados con depresión infantil

\begin{tabular}{lcc}
\hline Contenido & $\mathbf{N}^{\mathbf{0}}$ & documentos \% \\
\hline Depresión y adolescencia & 6 & 18 \\
Depresión y rendimiento académico & 4 & 12 \\
Depresión infantil y otros (autoestima, & & 53 \\
sintomatología, factores de riesgo, adolescencia, etc.) & 18 & 3 \\
Depresión en un colegio & 1 & 3 \\
Depresión & 1 & 8 \\
Prevención y/o terapia & 3 & 3 \\
Tristeza y Música & 1 & 100 \\
Total & 34 & \\
\hline
\end{tabular}

Fuente: Elaboración propia.

\section{DISCUSIÓN}

Realizando un análisis de contenido en la revisión, se muestra que existe una correlación entre la depresión y el rendimiento académico. En un estudio realizado en México por Robles y otros (2011) se señala que la depresión se presenta con más frecuencia en edades tempranas, a excepción del sexo femenino que tiene una elevada presencia en la adolescencia. En relación, al rendimiento académico, se 
registra mejor aprovechamiento académico en las mujeres que en los varones, y con respecto a las edades cuando más avanza ésta, son menores las calificaciones escolares. Además, los resultados arrojan que cuando el nivel de depresión es medio o bajo, el rendimiento escolar es más alto, sin embargo, es menor cuando la depresión es moderada y alta.

Otro estudio realizado en España con una muestra de 658 niños concluye que tienen bajo rendimiento académico aquellos que presentan síntomas de depresión. También, menciona que teniendo en cuenta el sexo, en los niños hay menor prevalencia de sintomatología depresiva que en las niñas (Orgilés, Gómez, Piqueras y Espada, 2014). En Argentina se realizó una investigación sobre la depresión en adolescentes y el desempeño académico, en el que se obtuvieron resultados débiles en los niveles de correlación, No obstante, al tener en cuenta variables como el sexo, la edad y el tipo de escuela (privada y pública), se encontró que la asociación es frecuente en mujeres, y en alumnos de colegios estatales. Esto quiere decir, que las adolescentes que presentan depresión suelen tener un bajo rendimiento académico.

Por otro lado, se reveló que las mujeres necesitan un mayor tratamiento en la prevención de este trastorno, ya que son más vulnerables a presentarlo (Pérez y Urquijo, 2001).

Asimismo, los resultados determinaron la mayor prevalencia de depresión en varones con un $16,3 \%$, mientras que las mujeres obtuvieron un $8,7 \%$. Esto llama la atención porque la mayoría de los estudios revisados y existentes muestran lo contrario. Además, la investigación no encontró relación entre depresión y rendimiento académico. Por lo tanto, se atribuye la causa de la depresión infantil a otros eventos ambientales y personales como condición genética, características psicológicas, problemática familiar, etc. (Moreno, Escobar, Vera, Calderón y Villamizar, 2009).

De las investigaciones revisadas se puede señalar que hay una presencia predominante de DI en las mujeres, se presenta en edades tempranas y se incrementa en la adolescencia. Por lo tanto, se debe conducir a un enfoque preventivo de la depresión infantil, y trabajar para fortalecer en edades primarias, sobre todo en el sexo femenino que muestra más vulnerabilidad frente a este. Tomando estrategias y programas que favorezcan en todos los aspectos el desarrollo saludable de los niños se disminuirá el índice de presencia de DI en los siguientes años.

El factor predominante de la depresión infantil es el pensamiento negativo. En los estudios revisados se encuentran otras causas clasificadas en dos grupos: personales y ambientales. El ser humano puede verse afectado por diversos estímulos de su propio entorno. El grado de vulnerabilidad está más relacionado e inclinado por la condición personal y psicológica, pero esta no condiciona su capacidad de adaptarse ante múltiples realidades adversas. Todos los estudios hacen énfasis en que el factor predominante es el personal relacionado al componente psicológico y específicamente a la formulación de pensamientos negativos con los cuales el niño interpreta su entorno. Estos pensamientos podrían inclinarlo para ponerlo en riesgo. 


\section{CONCLUSIONES}

Se puede concluir que el tema Depresión Infantil ha sido abordado por diversos estudios, pero aún no con la relevancia que se merece, a pesar de ser una de las causas más importantes de la mortalidad infantil en la actualidad. Asimismo, esta revisión debe llevar a la comunidad profesional peruana a realizar mayores investigaciones de campo en diversas poblaciones del país con el objetivo de seguir esclareciendo los factores predominantes de la depresión infantil en la cultura peruana.
En la revisión de este tema se han encontrado escasas investigaciones que lo aborden desde un enfoque preventivo, siendo necesario tomar iniciativas que cultiven una cultura de prevención ante la DI, que además de diagnósticos o tratamientos se concretice en la elaboración de programas y estrategias. Es importante y necesario prevenir para salvaguardar la salud mental de los niños, protegiendo su condición vulnerada por tantos conflictos en las familias de hoy en día. 


\section{REFERENCIAS}

Del Barrio, V. (2010). La depresión infantil a la altura de nuestro tiempo. Información psicológica, 100, 49-59.

Esparza, N. y Rodríguez, M.C. (2009). Factores contextuales del desarrollo infantil y su relación con los estados de ansiedad y depresión. Diversitas, 5(1), 47-65.

García-Vera, M. y Sanz, J. (2012). Depresión. Manual de terapia de conducta en la infancia. Madrid, España: Dykinson.

Grupo de trabajo de la actualización de la Guía de Práctica Clínica sobre la Depresión Mayor en la Infancia y la Adolescencia (2018). Guía de Práctica Clínica sobre la Depresión Mayor en la Infancia y la Adolescencia. Actualización. Ministerio de Sanidad, Servicios Sociales e Igualdad. Unidad de Asesoramiento Científico-técnico (Avalia-t); Guías de Práctica Clínica en el SNS. Recuperado de http://www.guiasalud. es/GPC/GPC_575_Depresion_infancia_Avaliat_compl.pdf

Huamanttica, R. (2016). Depresión infantil: Un problema desconocido por la sociedad peruana. Cultura, 30, 253-259. Recuperado de https://www.revistacultura.com.pe/ revistas/RCU_30_depresion-infantil-un-problema-desconocido-por-la-sociedadperuana.pdf

Kovacs, M. (2010). Prodromal symptoms and atypical affectivity as predictors of major depression in juveniles: Implications for prevention. Journal of Child Psychology and Psychiatry, 51, 472-496. doi: 10.1111/j.1469- 7610.2010.02230.x

Méndez, F. (2002). El niño que no sonríe. Estrategias para superar la tristeza y depresión infantil. Recuperado de https://serproductivo.org/wp-content/uploads/2017/08/Elni\%C3\%B1o-que-no-sonr\%C3\%ADe.pdf

Moreno, J., Escobar, A., Vera, A., Calderón, T. y Villamizar, L. (2009). Asociación entre depresión y rendimiento académico en un grupo de niños de la localidad de Usaquén. Psychologia. Avances de la disciplina, 3(1), 131-156. Recuperado de http://www. redalyc.org/articulo.oa? $\mathrm{id}=297225173007$

Organización Mundial de la Salud (2014). Health for the world's adolescents: a second chance in the second decade. Recuperado de http://apps.who.int/adolescent /seconddecade/files/1612_MNCAH_HWA_Executive_Summary.

Orgilés, M., Gómez, M., Piqueras, J., y Espada, J. (2014). Relación entre el rendimiento escolar y la sintomatología depresiva en niños españoles. Electronic Journal of Research in Educational Psychology, 12(33), 447 - 460. doi: 10.14204/ejrep.33.14009

Ortega, M. (2015). Factores de riesgo y protección de la depresión infantil: una revisión bibliográfica (Trabajo de Fin de Grado, Universidad de Jaén, España). Recuperada de http:/tauja.ujaen.es/bitstream/10953.1/1962/1/Ortega_Osorio_Macarena_TFG_ Psicologa.pdf

Paelecke, Y., Pohl, J., \& Leplow B. (2005). Attention and executive functions in remitted major depression patients. Journal of Affective Disorders, 89 (1-3), 125-135. 
Pérez, J., Rivera, C., Soto-Vega, E. y Muñoz-Pérez, M. (2011). Trastorno depresivo mayor en niños y adolescentes. Revista Mexicana Neurociencia, 12(3), 149 -154. Recuperado de https://www.researchgate.net/profile/Maria-Munoz-Perez-2/ publication/236680377_Trastorno_depresivo_mayor_en_ninos_y_adolescentes/ links/0c960518f325c72945000000/Trastorno-depresivo-mayor-en-ninos-yadolescentes.pdf

Pérez, M. y Urquijo, S. (2001). Depresión en adolescentes. Relaciones con el desempeño académico. Psicología Escolar e Educacional, 5(1), 49-58. Recuperado de http://pepsic. bvsalud.org/scielo.php?script=sci_arttext\&pid=S1413-85572001000100006\#end1

Pinto, B. y Álvarez, E. (2010). Terapia breve y depresión infantil. Revista Ajayu 8(1), 87106.

Ramírez, R. (2009). Adaptación del inventario de depresión infantil de Kovacs en escolares de Cajamarca (Tesis de Licenciatura en Psicología, Pontificia Universidad Católica del Perú, Lima). Recuperada de http://tesis.pucp.edu.pe/repositorio/bitstream/ handle/20.500.12404/409/RAMIREZ_BARRANTES_RENATO_ADAPTACION_ INVENTARIO_DEPRESION.pd??sequence $=1 \&$ isAllowed $=\mathrm{y}$

Robles, F., Sánchez, A., Galicia, I. (2011). Relación del bienestar psicológico, depresión y rendimiento académico en estudiantes de secundaria. Psicología Iberoamericana, 19 (2), 27-37. Recuperado de http://www.redalyc.org/html/1339/133921440004/

Siabato, E., Forero, I. y Salamanca, Y. (2017). Asociación entre depresión e ideación suicida en un grupo de adolescentes colombianos. Pensamiento Psicológico, 15(1), 51-61.doi:10.11144/Javerianacali.PPSI15-1.ADIS

Fecha de recepción: 28-11-2018

Fecha de aceptación: 16-08-2019 